\title{
Count-Invariance Including Exponentials
}

\author{
Stepan Kuznetsov \\ Steklov Mathematical Institute \\ (Moscow), RAS \\ sk@mi.ras.ru
}

\author{
Glyn Morrill \\ Universitat Politècnica \\ de Catalunya \\ morrill@cs.upc.edu
}

\author{
Oriol Valentín \\ Universitat Politècnica \\ de Catalunya \\ oriol.valentin@gmail.com
}

\begin{abstract}
We define infinitary count-invariance for categorial logic, extending countinvariance for multiplicatives (van Benthem, 1991) and additives and bracket modalities (Valentín et al., 2013) to include exponentials. This provides an effective tool for pruning proof search in categorial parsing/theorem-proving.
\end{abstract}

\section{Introduction}

In logical grammar, which dates back to (Ajdukiewicz, 1935), grammar is reduced to logic: an expression is grammatical if and only if an associated logical statement is a theorem of a calculus.

\subsection{Sharing}

In standard logic information does not have multiplicity. Thus where + is the notion of addition of information and $\leq$ is the notion of inclusion we have $x+x \leq x$ and $x \leq x+x$; both these two properties together amount to idempotency: $x+x=x$. These properties are expressed by the rules of inference of Contraction and Expansion:

(1) a. $\frac{\Delta(A, A) \Rightarrow B}{\Delta(A) \Rightarrow B}$ Contraction

$$
\text { b. } \frac{\Delta(A) \Rightarrow B}{\Delta(A, A) \Rightarrow B} \text { Expansion }
$$

Linguistic resources do not freely have these properties: grammaticality is not generally preserved under addition or removal of copies of words or expressions. However, there are some constructions manifesting something similiar. Parasitic gaps involve a kind of Contraction. Parasitic gaps cannot occur anywhere, thus:

(2) *the slave that ${ }_{i}$ John sold $e_{i}$ to $e_{i}$
Rather, we assume here that as the term 'parasitic' suggests, a parasitic gap must fall within an island. Extraction from weak islands can become fully acceptable when accompanied by a cobound non-island extraction:
(3) a. man that ${ }_{i}$ [the friends of $e_{i}$ ] admire $e_{i}$
b. paper that ${ }_{i}$ John filed $e_{i}$ [without reading $e_{i}$ ]
c. paper that ${ }_{i}$ [the editor of $e_{i}$ ] filed $e_{i}$ [without reading $e_{i}$ ]

And iterated coordination allows a kind of Expansion:

(4) John likes, Mary dislikes and Bill loves London.

That is, in logical grammar a controlled use of idempotency, or sharing, is motivated. Girard (1987) introduced exponentials for such control. Versions of the exponentials have been used to treat (parasitic) gaps and iterated coordination and iterated "respectively" in categorial grammar (Morrill, 2017), (Morrill and Valentín, 2015a, 2016b).

\subsection{Count-Invariance}

Count-invariance for multiplicatives in (sub)linear logic is introduced in van Benthem (1991). This involves simply checking the number of positive and negative occurrences of each atom in a sequent. Thus where $\#(\Sigma)$ is a count of the sequent $\Sigma$ we have:

$$
\text { (5) } \vdash \Sigma \Longrightarrow \#(\Sigma)=0
$$

I.e. the numbers of positive and negative occurrences of each atom must exactly balance for the sequent to be a theorem. This provides a necessary, but of course not sufficient, criterion for theoremhood, and can be checked rapidly. It can be 
used as a filter in proof search: if backward chaining proof search generates a goal which does not satisfy the count-invariant, the goal can be discarded. This notion of count for multiplicatives was included in the categorial parser/theoremprover CatLog (Morrill, 2012).

In Valentín et al. (2013) the idea is extended to additives (and bracket modalities). Instead of a single count for each atom of a sequent $\Sigma$ we have a minimum count $\#_{\min }(\Sigma)$ and a maximum count $\#_{\max }(\Sigma)$ and for a sequent to be a theorem it must satisfy two inequations:

(6) $\vdash \Sigma \Longrightarrow \#_{\min }(\Sigma) \leq 0 \leq \#_{\max }(\Sigma)$

I.e. the count functions $\#_{\min }$ and $\#_{\max }$ define an interval which must include the point of balance 0 ; for the multiplicatives, $\#_{\min }=\#_{\max }=\#$ and (6) reduces to the special case (5). This generalised notion of count is included in the categorial parser/theorem-prover CatLog2.

The structure of the continuation of the paper is as follows. In Section 2 we present the infinitary count algebra which we employ, we define the fragment of categorial logic for which we illustrate count invariance, and we define the (infinitary) count functions for this fragment. In Section 3 we state and prove our count-invariance theorem. In Section 4 we evaluate the introduction of exponential count invariance experimentally in relation to CatLog parsing/theorem-proving.

\section{Infinitary Count Algebra}

We consider terms built over constants 0,1 , $\perp(-\infty$ : minus infinity), and $T$ ( $+\infty$ : plus infinity) by binary operations of plus $(+)$, minus $(-)$, minimum (min) and maximum (max), and the infinitary step functions $X$ and $Y$ as follows where $i$ and $j$ are integers (* indicates undefined):

\begin{tabular}{c|ccc}
+ & $j$ & $\perp$ & $\top$ \\
\hline$i$ & $i+j$ & $\perp$ & $\top$ \\
$\perp$ & $\perp$ & $\perp$ & $*$ \\
$\top$ & $\top$ & $*$ & $\top$ \\
- & $j$ & $\perp$ & $\top$ \\
\hline$i$ & $i-j$ & $\top$ & $\perp$ \\
$\perp$ & $\perp$ & $*$ & $\perp$ \\
$\top$ & $\top$ & $\top$ & $*$
\end{tabular}

\begin{tabular}{c|ccc}
$\min$ & $j$ & $\perp$ & $\mathrm{T}$ \\
\hline$i$ & $\frac{|i+j|-|i-j|}{2}$ & $\perp$ & $i$ \\
$\perp$ & $\perp$ & $\perp$ & $\perp$ \\
$\mathrm{T}$ & $j$ & $\perp$ & $\mathrm{T}$
\end{tabular}

\begin{tabular}{c|ccc}
$\max$ & $j$ & $\perp$ & $\mathrm{\top}$ \\
\hline$i$ & $\frac{|i+j|+|i-j|}{2}$ & $i$ & $\mathrm{\top}$ \\
$\perp$ & $j$ & $\perp$ & $\mathrm{\top}$ \\
$\mathrm{\top}$ & $\mathrm{\top}$ & $\mathrm{\top}$ & $\mathrm{\top}$
\end{tabular}

$$
\begin{aligned}
& X(i)= \begin{cases}\top & \text { if } i>0 \\
i & \text { if } i \leq 0\end{cases} \\
& Y(i)= \begin{cases}i & \text { if } i \geq 0 \\
\perp & \text { if } i<0\end{cases}
\end{aligned}
$$

(7) Proposition. 0. $\perp<i<\mathrm{T}$; 1. for $a, b<\mathrm{T}$, $a+b<\mathrm{T} ; 2$. for $a, b>\perp, a+b>\perp ; 3$. for $a>\perp \& b<\mathrm{T}, b-a<\mathrm{T}$; 4 . for $b>\perp \& a<$ $\mathrm{T}, b-a>\perp ; 5$. for $a, b>\perp, \min (a, b)>\perp \&$ $\max (a, b)>\perp ; 6$. for $a, b<\mathrm{T}, \min (a, b)<\mathrm{T}$ $\& \max (a, b)<\mathrm{T} ; 7$. for $a>\perp, X(a)>\perp ; 8$. for $a<\mathrm{T}, Y(a)<\mathrm{T}$.

\subsection{The count functions}

The count function, or count functions, are functions from types and sequents into values in the count algebra such that if sequents are provable their images under the count functions fall within a certain range. It follows that if their images do not fall within the required range then the sequents are not provable; we give examples after defining the count functions, in the next subsection. This provides an efficient filter on parsing/theoremproving, as we show in the last section.

Let us assume primitive types $\mathcal{P}$. For $Q \in$ $\mathcal{P} \cup\{[]\}, m \in\{\min , \max \}$ and $\overline{\min }=\max$ and $\overline{\max }=\min$ we define

$$
\#_{m, Q}(\Gamma \Rightarrow A)=\#_{m, Q}^{\circ}(A)-\#_{\bar{m}, Q}^{\bullet}(\Gamma)
$$

where $\#^{\circ}$ and $\#^{\bullet}$ are as below. We define the enrichment $\mathbf{L A b} !_{\mathbf{b}}$ ? of the Lambek calculus (Lambek, 1958) with types Tp as follows:

$$
\begin{aligned}
\mathbf{T p}::= & \mathcal{P} \mid \\
& \mathbf{T p} \backslash \mathbf{T p}|\mathbf{T p} / \mathbf{T p}| \mathbf{T p} \bullet \mathbf{T p} \mid \\
& \mathbf{T p} \& \mathbf{T} \mathbf{p}|\mathbf{T} \mathbf{p} \oplus \mathbf{T} \mathbf{p}| \\
& {[]^{-1} \mathbf{T p}|\langle\rangle \mathbf{T p}| } \\
& ! \mathbf{T p} \mid ? \mathbf{T} \mathbf{p}
\end{aligned}
$$

Where $P \in \mathcal{P}, p \in\{\bullet, \circ\}$, and $\bar{\bullet}=\circ$ and $\bar{\circ}=\bullet$ we 
define the count functions:

$$
\begin{aligned}
\#_{m, Q}^{p}(P) & =1 \text { if } Q=P \\
\#_{m, Q}^{p}(A \backslash C) & =\#_{m, Q}^{p}(C)-\#_{\bar{m}, Q}^{\bar{p}}(A) \\
\#_{m, Q}^{p}(C / B) & =\#_{m, Q}^{p}(C)-\#_{\bar{p}, Q}^{\bar{p}}(B) \\
\#_{m, Q}^{p}(A \bullet B) & =\#_{m, Q}^{p}(A)+\#_{m, Q}^{p}(B) \\
\#_{m, Q}^{\circ}(A \& B) & =\bar{m}\left(\#_{m, Q}^{\circ}(A), \#_{m, Q}^{\circ}(B)\right) \\
\#_{m, Q}^{\bullet}(A \& B) & =m\left(\#_{m, Q}^{\bullet}(A), \#_{m, Q}^{\bullet}(B)\right) \\
\#_{m, Q}^{\circ}(A \oplus B) & =m\left(\#_{m, Q}^{\circ}(A), \#_{m, Q}^{\circ}(B)\right) \\
\#_{m, Q}^{\bullet}(A \oplus B) & \left.=\bar{m} \#_{m, Q}^{\bullet}(A), \#_{m, Q}^{\bullet}(B)\right) \\
\#_{m, P}^{p}\left([]^{-1} A\right) & =\#_{m, P}^{p}(A) \\
\#_{m,[]}^{p}\left([]^{-1} A\right) & =\#_{m,[]}^{p}(A)-1 \\
\#_{m, P}^{p}(\langle\rangle A) & =\#_{m, P}^{p}(A) \\
\#_{m,[]}^{p}(\langle\rangle A) & =\#_{m,[]}^{p}(A)+1 \\
\#_{m, Q}^{\circ}(! A) & =\#_{m, Q}^{\circ}(A) \\
\#_{\min , Q}^{\bullet}(! A) & =Y\left(\#_{\min , Q}^{\bullet}(A)\right) \\
\#_{\max , P}^{\bullet}(! A) & =X\left(\#_{\max , P}^{\bullet}(A)\right) \\
\#_{\max ,[]}^{\bullet}(! A) & =\top \\
\#_{\min , Q}^{\circ}(? A) & =Y\left(\#_{\min , Q}^{\circ}(A)\right) \\
\#_{\max , Q}^{\circ}(? A) & =X\left(\#_{\max , Q}^{\circ}(A)\right) \\
\#_{m, Q}^{\bullet}(? A) & =\#_{m, Q}^{\bullet}(A)
\end{aligned}
$$

(8) Lemma. $\#_{m}^{p}(A)$ is defined and $\perp<$ $\#_{\max }(A) \& \#_{\min }(A)<\mathrm{T}$.

Proof. By induction as in Figure 1; justifications refer to the Proposition (7).

To present sequents we define configurations Config and tree terms TreeTerm in terms of types $\mathbf{T p}$ as follows, where $\Lambda$ is the empty string:

\section{Config $::=\Lambda \mid$ TreeTerm, Config TreeTerm ::= Tp| $\mid$ Config $]$}

The rules for $\mathbf{L A b} !_{\mathbf{b}}$ ? are shown in Figure 2. Note that $! C$ is of a generalised form necessary to prove Cut-elimination in the presence of $! R$. Note also that $? L$ is an infinitary rule; it is not used in linguistic applications. We include it here for the sake of showing technical completeness of the count invariance. For tree terms and configurations, counts are:

$$
\begin{aligned}
\#_{m, Q}^{\bullet}(\Gamma, \Delta) & =\#_{m, Q}^{\bullet}(\Gamma)+\#_{m, Q}^{\bullet}(\Delta) \\
\#_{m, P}^{\bullet}([\Gamma]) & =\#_{m, P}(\Gamma) \\
\#_{m,[]}^{\bullet}([\Gamma]) & =\#_{m,[]}^{\bullet}(\Gamma)+1 \\
\#_{m, Q}^{\bullet}(\Lambda) & =0
\end{aligned}
$$

Lemma 8 extends to configurations.

\subsection{Examples}

Relativisation including medial and parasitic extraction is obtained by assigning a relative pronoun a type $(C N \backslash C N) /(! N \backslash S)$ whereby the body of a relative clause is analysed as $! N \backslash S$. By way of example of count-invariance, we show how it discards $N, N \backslash S \Rightarrow ! N \backslash S$ corresponding to the ungrammaticality of a relative clause without a gap: *paper that John walks. We have the max $N$-count: $\#_{\max , N}(N, N \backslash S \Rightarrow ! N \backslash S)=$ $\#_{\max , N}^{\circ}(! N \backslash S)-\#_{\min , N}^{\bullet}(N, N \backslash S)=\#_{\max . N}^{\circ}(S)-$ $\#_{\min , N}^{\bullet}(! N)-\#_{\min , N}^{\bullet}(N)-\#_{\min , N}^{\bullet}(N \backslash S)=0-$ $Y\left(\#_{\min , N}^{\bullet}(N)\right)-1-\#_{\min , N}^{\bullet}(S)+\#_{\min , N}^{\mathrm{O}}(N)=$ $-Y(1)-1-0+1=-1-1+1=-1 \nsupseteq 0$ which means that the count-invariance is not satisfied.

Iterated sentential coordination is obtained by assigning a coordinator the type $(? S \backslash S) / S$. By way of a second example we show how count-invariance discards $N, N, N \backslash S \Rightarrow ? S$ corresponding to the ungrammaticality of unequilibrated coordination: *John Mary walks and Suzy talks. Max $N$-count is: $\#_{\max , N}(N, N, N \backslash S \Rightarrow ? S)=\#_{\max , N}^{\circ}(? S)-$ $\# \bullet_{\min , N}(N, N, N \backslash S)=X\left(\#_{\max , N}^{\circ}(S)\right) \quad-$ $\#_{\min , N}^{\bullet}(N)-\#_{\min , N}^{\bullet}(N)-\#_{\min , N}^{\bullet}(N \backslash S)=$ $X(0)-1-1-\#_{\min , N}^{\bullet}(S)+\#_{\max , N}^{\bullet}(N)=$ $0-2-0+1=-1 \nsupseteq 0$ which means that the count-invariance is not satisfied.

\section{Theorem and Proof}

Our main theorem is:

\section{(9) Theorem.}

$$
\begin{aligned}
& \vdash \Gamma \Rightarrow A \Longrightarrow \forall Q \in \mathcal{P} \cup\{[]\}, \\
& \#_{\min , Q}(\Gamma \Rightarrow A) \leq 0 \leq \#_{\max , Q}(\Gamma \Rightarrow A) \\
& \text { where as we have said, } \\
& \#_{m, Q}(\Gamma \Rightarrow A)=\#_{m, Q}^{\circ}(A)-\#_{\bar{m}, Q}^{\bullet}(\Gamma) .
\end{aligned}
$$

Proof. The proof is by induction on the length of derivations. For the base case $P \Rightarrow P$ we have $\#_{m, Q}(P \Rightarrow P)=\#_{m, Q}^{\circ}(P)-\#_{m, Q}^{\bullet}(P)=0$. The inductive cases are as follows, where we use:

- $a+b=b+a$

- $a+(b+c)=(a+b)+c$

- $a-(b+c)=(a-b)-c$ (including the undefined case)

- $(a+b)-c=(a-c)+b$ 


$$
\begin{aligned}
& \#_{m, Q}^{p}(P)=\begin{array}{ll}
1 & \text { if } Q=P \\
0 & \text { if } Q \neq P
\end{array} \\
& \#_{\max , Q}^{p}(A \backslash C)=\#_{\max , Q}^{p}(C)-\#_{\min , Q}^{\bar{p}}(A) \quad 4 \\
& \#_{\min , Q}^{p}(A \backslash C)=\#_{\min , Q}^{p}(C)-\#_{\max , Q}^{\bar{p}}(A) \\
& \#_{\max , Q}^{p}(C / B)=\#_{\max , Q}^{p}(C)-\#_{\min , Q}^{\bar{p}}(B) \quad 4 \\
& \#_{\min , Q}^{p}(C / B)=\#_{\min , Q}^{p}(C)-\#_{\max , Q}^{\bar{p}}(B) \\
& \#_{\max , Q}^{p}(A \bullet B)=\#_{\max , Q}^{p}(A)+\#_{\max , Q}^{p}(B) \quad 2 \\
& \#_{\min , Q}^{p}(A \bullet B)=\# p_{\min , Q}(A)+\# p_{\min , Q}(B) \quad 1 \\
& \#_{\min , Q}^{\circ}(A \& B)=\max \left(\#_{\min , Q}^{\circ}(A), \#_{\min , Q}^{\circ}(B)\right) 6 \\
& \#_{\max , Q}^{\circ}(A \& B)=\min \left(\#_{\max , Q}^{\circ}(A), \#_{\max , Q}^{\circ}(B)\right) 5 \\
& \#_{\min , Q}^{\bullet}(A \& B)=\min \left(\#_{\min , Q}^{\bullet}(A), \#_{\min , Q}^{\bullet}(B)\right) \quad 6 \\
& \#_{\max , Q}^{\bullet}(A \& B)=\max \left(\#_{\max , Q}^{\bullet}(A), \#_{\max , Q}^{\bullet}(B)\right) 5 \\
& \#_{\min , Q}^{\circ}(A \oplus B)=\min \left(\#_{\min , Q}^{\circ}(A), \#_{\min , Q}^{\circ}(B)\right) 6 \\
& \#_{\max , Q}^{\circ}(A \oplus B)=\max \left(\#_{\max , Q}^{\circ}(A), \#_{\max , Q}^{\circ}(B)\right) 5 \\
& \#_{\min , Q}^{\bullet}(A \oplus B)=\max \left(\#_{\min , Q}^{\bullet}(A), \#_{\min , Q}^{\bullet}(B)\right) \quad 6 \\
& \#_{\max , Q}^{\bullet}(A \oplus B)=\min \left(\#_{\max , Q}^{\bullet}(A), \#_{\max , Q}^{\bullet}(B)\right) 5 \\
& \#_{\max , P}^{p}\left([]^{-1} A\right)=\#_{\max , P}^{p}(A)>\perp \\
& \#_{\min , P}^{P}\left([]^{-1} A\right)=\#_{\min , P}^{p}(A)<\mathrm{T} \\
& \#_{\max ,[]}^{p}\left([]^{-1} A\right)=\#_{\max ,[]}^{p}(A)-1>\perp \\
& \#_{\min ,[]}^{p}\left([]^{-1} A\right)=\#_{\min ,[]}^{p}(A)-1<\mathrm{T} \\
& \#_{\max , P}^{p}(\langle\rangle A)=\#_{\max , P}^{p}(A)>\perp \\
& \#_{\min , P}^{p}(\langle\rangle A)=\#_{\min , P}^{p}(A)<\perp \\
& \#_{\max ,[]}^{p}(\langle\rangle A)=\#_{\max ,[]}^{p}(A)+1>\perp \\
& \#_{\min ,[]}^{p}(\langle\rangle A)=\#_{\min ,[]}^{p}(A)+1<\mathrm{T} \\
& \#_{\max , P}^{\bullet}(! A)=X\left(\#_{\max , P}^{\bullet}(A)\right) \\
& \#_{\min , Q}^{\bullet}(! A)=Y\left(\#_{\min , Q}^{\bullet}(A)\right) \\
& \#_{\max ,[]}^{\bullet}(! A)=\top>\perp \\
& \#_{\max , Q}^{\circ}(! A)=\#_{\max , Q}^{\circ}(A)>\perp \\
& \#_{\min , Q}^{\circ}(! A)=\#_{\min , Q}^{\circ}(A)<\mathrm{T} \\
& \#_{\max , Q}^{\circ}(? A)=X\left(\#_{\max , Q}^{\circ}(A)\right) \\
& \#_{\min , Q}^{\circ}(? A)=Y\left(\#_{\min , Q}^{\circ}(A)\right) \\
& \#_{\min , Q}^{\bullet}(? A)=\#_{\min , Q}^{\bullet}(A)<\mathrm{T} \\
& \#_{\max , Q}^{\bullet}(? A)=\#_{\max , Q}^{\bullet}(A)>\perp
\end{aligned}
$$

(




$$
\begin{aligned}
& \overline{P \Rightarrow P} i d, P \in \mathcal{P} \\
& \frac{\Gamma \Rightarrow A \quad \Delta(C) \Rightarrow D}{\Delta(\Gamma, A \backslash C) \Rightarrow D} \backslash L \quad \frac{\Gamma \Rightarrow B \quad \Delta(C) \Rightarrow D}{\Delta(C / B, \Gamma) \Rightarrow D} \backslash L \\
& \frac{A, \Gamma \Rightarrow C}{\Gamma \Rightarrow A \backslash C} \backslash R \quad \frac{\Gamma, B \Rightarrow C}{\Gamma \Rightarrow C / B} / R \\
& \frac{\Delta(A, B) \Rightarrow C}{\Delta(A \bullet B) \Rightarrow C} \bullet L \quad \frac{\Gamma_{1} \Rightarrow A \quad \Gamma_{2} \Rightarrow B}{\Gamma_{1}, \Gamma_{2} \Rightarrow A \bullet B} \bullet R \\
& \frac{\Delta(A) \Rightarrow D}{\Delta(A \& B) \Rightarrow D} \& L_{1} \quad \frac{\Delta(B) \Rightarrow D}{\Delta(A \& B) \Rightarrow D} \& L_{2} \quad \frac{\Gamma \Rightarrow A \quad \Gamma \Rightarrow B}{\Gamma \Rightarrow A \& B} \& R \\
& \frac{\Gamma \Rightarrow A}{\Gamma \Rightarrow A \oplus B} \oplus R_{1} \quad \frac{\Gamma \Rightarrow B}{\Gamma \Rightarrow A \oplus B} \oplus R_{2} \quad \frac{\Delta(A) \Rightarrow D \quad \Delta(B) \Rightarrow D}{\Delta(A \oplus B) \Rightarrow D} \oplus L \\
& \frac{\Gamma(A) \Rightarrow B}{\Gamma\left(\left[[]^{-1} A\right]\right) \Rightarrow B}[]^{-1} L \quad \frac{[\Gamma] \Rightarrow A}{\Gamma \Rightarrow[]^{-1} A}[]^{-1} R \\
& \frac{\Gamma([A]) \Rightarrow B}{\Gamma(\langle\rangle A) \Rightarrow B}\langle\rangle L \quad \frac{\Gamma \Rightarrow A}{[\Gamma] \Rightarrow\langle\rangle A}\langle\rangle R \\
& \frac{\Delta(A) \Rightarrow D}{\Delta(! A) \Rightarrow D} ! L \quad \frac{! A_{1}, \ldots, ! A_{n} \Rightarrow A}{! A_{1}, \ldots, ! A_{n} \Rightarrow ! A} ! R \\
& \frac{\Delta(\Gamma, ! A) \Rightarrow D}{\Delta(! A, \Gamma) \Rightarrow D} ! P_{1} \quad \frac{\Delta(! A, \Gamma) \Rightarrow D}{\Delta(\Gamma, ! A) \Rightarrow D} ! P_{2} \quad \frac{\Delta\left(! A_{0}, \ldots, ! A_{n},\left[! A_{0}, \ldots, ! A_{n}, \Gamma\right]\right) \Rightarrow D}{\Delta\left(! A_{0}, \ldots, ! A_{n}, \Gamma\right) \Rightarrow D} ! C \\
& \frac{\Delta(A) \Rightarrow D \quad \Delta(A, A) \Rightarrow D \quad \cdots}{\Delta(? A) \Rightarrow D} ? L \quad \frac{\Gamma \Rightarrow A}{\Gamma \Rightarrow ? A} ? R \\
& \frac{\Gamma_{1} \Rightarrow C \quad \Gamma_{2} \Rightarrow ? C}{\Gamma_{1}, \Gamma_{2} \Rightarrow ? C} ? M
\end{aligned}
$$

Figure 2: Rules for the categorial logic fragment $\mathbf{L A b} !_{\mathbf{b}}$ ? 
- $a-(b-c)=(a-b)+c$

(Where we write $\#(\Delta)$ with $\Delta$ a context we should more precisely understand that $\Delta$ is a configuration with a hole where the count of a hole is always zero.)

\section{Multiplicatives}

$$
\text { - } \frac{\Gamma \Rightarrow A \quad \Delta(C) \Rightarrow D}{\Delta(\Gamma, A \backslash C) \Rightarrow D} \backslash L
$$

For every atom or bracket,

$$
\begin{aligned}
& \#_{m}(\Delta(\Gamma, A \backslash C) \Rightarrow D)= \\
& \#_{m}^{\circ}(D)-\#_{\bar{m}}^{\bullet}(\Delta)-\#_{\bar{m}}^{\bullet}(\Gamma)-\#_{\bar{m}}^{\bullet}(A \backslash C)= \\
& \#_{m}^{\circ}(D)-\#_{\bar{m}}^{\bullet}(\Delta)-\#_{\bar{m}}^{\bullet}(\Gamma)-\#_{\bar{m}}^{\bullet}(C)+\#_{m}^{\circ}(A)= \\
& \#_{m}^{\circ}(A)-\#_{\bar{m}}^{\bullet}(\Gamma)+\#_{m}^{\circ}(D)-\#_{\bar{m}}^{\bullet}(\Delta)-\#_{\bar{m}}^{\bullet}(C)= \\
& \#_{m}(\Gamma \Rightarrow A)+\#_{m}(\Delta(C) \Rightarrow D)
\end{aligned}
$$

The induction hypothesis (i.h.) tells us that $\#_{\text {min }}(\Gamma \Rightarrow A) \leq 0$ and $\#_{\text {min }}(\Delta(C) \Rightarrow D) \leq 0$. Thus $\#_{\min }(\Delta(\Gamma, A \backslash C) \Rightarrow D)=\#_{\min }(\Gamma \Rightarrow A)+$ $\#_{\min }(\Delta(C) \Rightarrow D) \leq \quad 0 . \quad$ Similarly, $0 \leq \#_{\max }(\Delta(\Gamma, A \backslash C) \Rightarrow D)=\#_{\max }(\Gamma \Rightarrow A)+$ $\#_{\max }(\Delta(C) \Rightarrow D)$ by i.h. Therefore we have:

$$
\begin{aligned}
& \#_{\min }(\Delta(\Gamma, A \backslash C) \Rightarrow D) \leq 0 \leq \\
& \#_{\max }(\Delta(\Gamma, A \backslash C) \Rightarrow D)
\end{aligned}
$$

$$
\text { - } \frac{\Gamma \Rightarrow B \quad \Delta(C) \Rightarrow D}{\Delta(C / B, \Gamma) \Rightarrow D} \backslash L
$$

Like $\backslash L$.

$$
\text { - } \frac{A, \Gamma \Rightarrow C}{\Gamma \Rightarrow A \backslash C} \backslash R
$$

For every atom or bracket,

$$
\begin{aligned}
& \#_{m}(\Gamma \Rightarrow A \backslash C)= \\
& \#_{m}^{\circ}(A \backslash C)-\#_{\bar{m}}^{\bullet}(\Gamma)= \\
& \#_{m}^{\circ}(C)-\#_{\bar{m}}^{\bullet}(A)-\#_{\bar{m}}^{\bullet}(\Gamma)= \\
& \#_{m}(A, \Gamma \Rightarrow C)
\end{aligned}
$$

Therefore by i.h.,

$$
\begin{aligned}
& \#_{\min }(\Gamma \Rightarrow A \backslash C) \leq 0 \leq \#_{\max }(\Gamma \Rightarrow A \backslash C) \\
& \bullet \frac{\Gamma, B \Rightarrow C}{\Gamma \Rightarrow C / B} / R
\end{aligned}
$$

Like $\backslash R$.

- $\frac{\Delta(A, B) \Rightarrow C}{\Delta(A \bullet B) \Rightarrow C} \bullet L$

For every atom or bracket,

$$
\begin{aligned}
& \#_{m}(\Delta(A \bullet B) \Rightarrow C)= \\
& \#_{m}^{\circ}(C)-\#_{\bar{m}}^{\bullet}(\Delta)-\#_{\bar{m}}^{\bullet}(A \bullet B)= \\
& \#_{m}^{\circ}(C)-\#_{\bar{m}}^{\bullet}(\Delta)-\#_{\bar{m}}^{\bullet}(A)-\#_{\bar{m}}^{\bullet}(B)= \\
& \#_{m}(\Delta(A, B) \Rightarrow C)
\end{aligned}
$$

Therefore by i.h.,

$$
\begin{gathered}
\#_{\min }(\Delta(A \bullet B) \Rightarrow C) \leq 0 \leq \\
\#_{\max }(\Delta(A \bullet B) \Rightarrow C) \\
\bullet \frac{\Gamma_{1} \Rightarrow A \quad \Gamma_{2} \Rightarrow B}{\Gamma_{1}, \Gamma_{2} \Rightarrow A \bullet B} \bullet R
\end{gathered}
$$

For every atom or bracket,

$$
\begin{aligned}
& \#_{m}\left(\Gamma_{1}, \Gamma_{2} \Rightarrow A \bullet B\right)= \\
& \#_{m}^{\circ}(A \bullet B)-\#_{\bar{m}}^{\bullet}\left(\Gamma_{1}, \Gamma_{2}\right)= \\
& \#_{m}^{\circ}(A)-\#_{\bar{m}}^{\bullet}\left(\Gamma_{1}\right)+\#_{m}^{\circ}(B)-\#_{\bar{m}}^{\bullet}\left(\Gamma_{2}\right)= \\
& \#_{m}\left(\Gamma_{1} \Rightarrow A\right)+\#_{m}\left(\Gamma_{2} \Rightarrow B\right)
\end{aligned}
$$

Therefore by i.h.,

$$
\#_{\min }\left(\Gamma_{1}, \Gamma_{2} \Rightarrow A \bullet B\right) \leq 0 \leq
$$$$
\#_{\max }\left(\Gamma_{1}, \Gamma_{2} \Rightarrow A \bullet B\right)
$$

\section{Additives}

$$
\text { - } \frac{\Delta(A) \Rightarrow D}{\Delta(A \& B) \Rightarrow D} \& L_{1}
$$

For every atom or bracket,

$$
\begin{aligned}
& \#_{\min }(\Delta(A \& B) \Rightarrow D)= \\
& \#_{\min }^{\circ}(D)-\#_{\max }^{\bullet}(\Delta)-\#_{\max }^{\bullet}(A \& B)= \\
& \#_{\min }^{\circ}(D)-\#_{\max }^{\bullet}(\Delta)- \\
& \max \left(\#_{\max }^{\bullet}(A), \#_{\max }^{\bullet}(B)\right) \leq \\
& \#_{\min }^{\circ}(D)-\#_{\max }^{\bullet}(\Delta)-\#_{\text {max }}^{\bullet}(A)= \\
& \#_{\min }(\Delta(A) \Rightarrow D) \leq 0 \text { i.h. }
\end{aligned}
$$


$\#_{\max }(\Delta(A \& B) \Rightarrow D)=$

$\#_{\max }^{\circ}(D)-\#_{\min }^{\bullet}(\Delta)-\#_{\min }^{\bullet}(A \& B)=$ $\#_{\max }^{\circ}(D)-\#_{\min }^{\circ}(\Delta)-$

$\min \left(\#_{\min }^{\bullet}(A), \#_{\min }^{\bullet}(B)\right) \geq$

$\#_{\text {max }}^{\circ}(D)-\#_{\text {min }}^{\bullet}(\Delta)-\#_{\text {min }}^{\bullet}(A)=$

$\#_{\max }(\Delta(A) \Rightarrow D) \geq 0$ i.h.

Therefore:

$\#_{\min }(\Delta(A \& B) \Rightarrow D) \leq 0 \leq$

$\#_{\max }(\Delta(A \& B) \Rightarrow D)$

- $\frac{\Delta(B) \Rightarrow D}{\Delta(A \& B) \Rightarrow D} \& L_{2}$

Like $\& L_{1}$

- $\frac{\Gamma \Rightarrow A \quad \Gamma \Rightarrow B}{\Gamma \Rightarrow A \& B} \& R$

$\#_{\min }(\Gamma \Rightarrow A \& B)=$

$\#_{\min }^{\circ}(A \& B)-\#_{\max }^{\bullet}(\Gamma)=$

$\max \left(\#_{\min }^{\circ}(A), \#_{\min }^{\circ}(B)\right)-\#_{\max }^{\bullet}(\Gamma)=$ $\max \left(\#_{\min }^{O}(A)-\#_{\max }^{\bullet}(\Gamma)\right.$,

$\left.\#_{\min }^{\circ}(B)-\#_{\max }^{\bullet}(\Gamma)\right)=$

$\underbrace{\max (\underbrace{\#_{\min }(\Gamma \Rightarrow A)}_{\leq 0 \text { i.h. }}, \underbrace{\#_{\min }(\Gamma \Rightarrow B)}_{\leq 0 \text { i.h. }})}_{\leq 0}$

And

$\#_{\max }(\Gamma \Rightarrow A \& B)=$

$\#_{\max }^{\circ}(A \& B)-\#_{\min }^{\bullet}(\Gamma)=$

$\min \left(\#_{\max }^{\circ}(A), \#_{\max }^{O}(B)\right)-\#_{\min }^{\bullet}(\Gamma)=$ $\min \left(\#_{\max }^{\circ}(A)-\#_{\min }^{\bullet}(\Gamma)\right.$,

$\left.\#_{\max }^{\circ}(B)-\#_{\min }^{\bullet}(\Gamma)\right)=$

$\min (\underbrace{\#_{\max }(\Gamma \Rightarrow)}_{\max }, \underbrace{\#_{\max }(\Gamma \Rightarrow B)}_{\max })$

$\underbrace{0 \leq i . h .}_{0 \leq \text { i.h. }}$

Therefore:

$$
\begin{aligned}
& \#_{\min }(\Gamma \Rightarrow A \& B) \leq 0 \leq \#_{\max }(\Gamma \Rightarrow A \& B) . \\
& \text { - } \frac{\Gamma \Rightarrow A}{\Gamma \Rightarrow A \oplus B} \oplus R_{1} \\
& \#_{\min }(\Gamma \Rightarrow A \oplus B)= \\
& \#_{\min }^{\circ}(A \oplus B)-\#_{\text {max }}^{\bullet}(\Gamma)= \\
& \min \left(\#_{\min }^{\circ}(A), \#_{\min }^{\bullet}(B)\right)-\#_{\max }^{\bullet}(\Gamma) \leq \\
& \#_{\min }^{\circ}(A)-\#_{\max }^{\bullet}(\Gamma)= \\
& \#_{\min }(\Gamma \Rightarrow A) \leq 0 \text { i.h. }
\end{aligned}
$$

And

$\#_{\max }(\Gamma \Rightarrow A \oplus B)=$

$\#_{\max }^{\circ}(A \oplus B)-\#_{\min }^{\bullet}(\Gamma)=$

$\max \left(\#_{\max }^{\bullet}(A), \#_{\max }^{\bullet}(B)\right)-\#_{\min }^{\bullet}(\Gamma) \geq$

$\#_{\max }^{\bullet}(A)-\#_{\min }^{\bullet}(\Gamma)=$

$\#_{\max }(\Gamma \Rightarrow A) \geq 0$ i.h.

- $\frac{\Gamma \Rightarrow B}{\Gamma \Rightarrow A \oplus B} \oplus R_{2}$

Like $\oplus R_{1}$.

$$
\text { - } \frac{\Delta(A) \Rightarrow D \quad \Delta(B) \Rightarrow D}{\Delta(A \oplus B) \Rightarrow D} \oplus L
$$

For every atom or bracket,

$$
\begin{aligned}
& \#_{\min }(\Delta(A \oplus B) \Rightarrow D)= \\
& \#_{\min }^{\circ}(D)-\#_{\text {max }}^{\bullet}(\Delta)-\#_{\text {max }}^{\bullet}(A \oplus B)= \\
& \#_{\min }^{\mathrm{O}}(D)-\#_{\max }^{\bullet}(\Delta)- \\
& \min \left(\#_{\max }^{\bullet}(A), \#_{\max }^{\bullet}(B)\right)= \\
& \max \left(\#_{\min }^{\circ}(D)-\#_{\max }^{\bullet}(\Delta)-\#_{\max }^{\bullet}(A)\right. \text {, } \\
& \left.\#_{\min }^{\circ}(D)-\#_{\max }^{\bullet}(\Delta)-\#_{\max }^{\bullet}(B)\right)= \\
& \underbrace{\max (\underbrace{\#_{\min }(\Delta(A) \Rightarrow D)}_{\leq 0 \text { i.h. }}, \underbrace{\#_{\min }(\Delta(B) \Rightarrow D)}_{\leq 0 \text { i.h. }})}_{\leq 0} \\
& 0 \leq \#_{\max }(\Delta(A \oplus B) \Rightarrow D) \text { similarly }
\end{aligned}
$$

\section{Bracket modalities}

$$
\text { - } \frac{\Gamma(A) \Rightarrow B}{\Gamma\left(\left[[]^{-1} A\right]\right) \Rightarrow B}[]^{-1} L
$$

For atoms:

$$
\begin{aligned}
& \#_{m, P}\left(\Gamma\left(\left[[]^{-1} A\right]\right) \Rightarrow B\right)= \\
& \#_{m, P}^{\circ}(B)-\#_{\bar{m}, P}^{\bullet}\left(\Gamma\left(\left[[]^{-1} A\right]\right)\right)= \\
& \left.\#_{m, P}^{\circ}(B)-\#_{\bar{m}, P}^{\bullet}(\Gamma)-\#_{\bar{m}, P}^{\bullet}\left(\left[[]^{-1} A\right]\right)\right)= \\
& \left.\#_{m, P}^{\circ}(B)-\#_{\bar{m}, P}^{\bullet}(\Gamma)-\#_{\bar{m}, P}^{\bullet}\left([]^{-1} A\right)\right)= \\
& \left.\#_{m, P}^{\circ}(B)-\#_{\bar{m}, P}^{\bullet}(\Gamma)-\#_{\bar{m}, P}^{\bullet}(A)\right)= \\
& \#_{m, P}^{\circ}(B)-\#_{\bar{m}, P}^{\bullet}(\Gamma(A))= \\
& \#_{m, P}(\Gamma(A) \Rightarrow B)
\end{aligned}
$$

I.e. the property for the conclusion follows from the induccion hypothesis for the premise since brackets and bracket modalities are transparent to atom count.

For brackets: 
$\#_{m,[]}\left(\Gamma\left(\left[[]^{-1} A\right]\right) \Rightarrow B\right)=$

$\#_{m,[]}^{\circ}(B)-\#_{\bar{m},[]}^{\bullet}\left(\Gamma\left(\left[[]^{-1} A\right]\right)\right)=$

$\left.\#_{m,[]}^{\circ}(B)-\#_{\bar{m},[]}^{\bullet}(\Gamma)-\#_{\bar{m},[]}^{\bullet}\left(\left[[]^{-1} A\right]\right)\right)=$

$\#_{m,[]}^{\circ}(B)-\#_{\bar{m},[]}^{\bullet}(\Gamma)-\#_{\bar{m},[]}^{\bullet}\left([]^{-1} A\right)-1=$

$\#_{m,[]}^{\circ}(B)-\#_{\bar{m},[]}^{\bullet}(\Gamma)-\#_{\bar{m},[]}^{\bullet}(A)+1-1=$

$\left.\#_{m,[]}^{\circ}(B)-\#_{\bar{m},[]}^{\bullet}(\Gamma)-\#_{\bar{m},[]}^{\bullet}(A)\right)=$

$\#_{m,[]}^{\circ}(B)-\#_{\bar{m},[]}^{\bullet}(\Gamma(A))=$

$\#_{m,[]}(\Gamma(A) \Rightarrow B)=$

Therefore by i.h.,

$\#_{\min }\left(\Gamma\left(\left[[]^{-1} A\right]\right) \Rightarrow B\right) \leq 0 \leq$

$\#_{\max }\left(\Gamma\left(\left[[]^{-1} A\right]\right) \Rightarrow B\right)$

$$
\text { • } \frac{[\Gamma] \Rightarrow A}{\Gamma \Rightarrow[]^{-1} A}[]^{-1} R
$$

For atoms:

$$
\begin{aligned}
& \#_{m, P}\left(\Gamma \Rightarrow[]^{-1} A\right)= \\
& \#_{m, P}(\Gamma \Rightarrow A)= \\
& \#_{m, P}([\Gamma] \Rightarrow A)
\end{aligned}
$$

Since brackets and bracket modalities are transparant to atom count.

For brackets:

$$
\begin{aligned}
& \#_{m,[]}\left(\Gamma \Rightarrow[]^{-1} A\right)= \\
& \#_{m,[]}^{\circ}\left([]^{-1} A\right)-\#_{\bar{m},[]}^{\bullet}(\Gamma)= \\
& \#_{m,[]}^{\circ}(A)-1-\#_{\bar{m},[]}^{\bullet}(\Gamma)= \\
& \#_{m,[]}^{\circ}(A)-\left(\#_{\bar{m},[]}^{\bullet}(\Gamma)+1\right)= \\
& \#_{m,[]}^{\circ}(A)-\#_{\bar{m},[]}^{\bullet}([\Gamma])= \\
& \#_{m,[]}([\Gamma] \Rightarrow A)
\end{aligned}
$$

Therefore by i.h.

$$
\begin{gathered}
\#_{\min }\left(\Gamma \Rightarrow[]^{-1} A\right) \leq 0 \leq \#_{\max }\left(\Gamma \Rightarrow[]^{-1} A\right) \\
\bullet \frac{\Gamma([A]) \Rightarrow B}{\Gamma(\langle\rangle A) \Rightarrow B}\langle\rangle L
\end{gathered}
$$

For atoms,

$$
\#_{m, P}(\Gamma(\langle\rangle A) \Rightarrow B)=\#_{m, P}(\Gamma([A]) \Rightarrow B)
$$

since brackets and bracket modalities are transparent to atom count.

For brackets,
$\#_{m,[]}(\Gamma(\langle\rangle A) \Rightarrow B)=$

$\#_{m,[]}^{o}(B)-\#_{\bar{m},[]}^{\bullet}(\Gamma)-\#_{\bar{m},[]}^{\bullet}(\langle\rangle A)=$

$\#_{m,[]}^{\circ}(B)-\#_{\bar{m},[]}^{\bullet}(\Gamma)-\left(\#_{\bar{m},[]}^{\bullet}(A)+1\right)=$

$\#_{m,[]}^{o}(B)-\#_{\bar{m},[]}^{\bullet}(\Gamma)-\#_{\bar{m},[]}^{\bullet,[}([A])=$

$\#_{m,[]}(\Gamma([A]) \Rightarrow B)$

Therefore by $i . h$.

$$
\#_{\min }\left(\Gamma(\langle>A) \Rightarrow B) \leq 0 \leq \#_{\max }(\Gamma(\langle>A) \Rightarrow B)\right.
$$

- $\frac{\Gamma \Rightarrow A}{[\Gamma] \Rightarrow\langle\rangle A}\langle\rangle R$

For atoms,

$\#_{m, P}([\Gamma] \Rightarrow\langle\rangle A)=\#_{m, P}(\Gamma \Rightarrow A)$

since brackets and bracket modalities are transparent to atom count.

For brackets,

$$
\begin{aligned}
& \#_{m,[]}([\Gamma] \Rightarrow\langle\rangle A)= \\
& \#_{m,[]}^{\circ}(\langle\rangle A)-\#_{\bar{m},[]}^{\bullet}([\Gamma])= \\
& \#_{m,[]}^{\circ}(A)+1-\#_{\bar{m},[]}^{\bullet}(\Gamma)-1= \\
& \#_{m,[]}^{\circ}(A)-\#_{\bar{m},[]}^{\bullet}(\Gamma)= \\
& \#_{m,[]}(\Gamma \Rightarrow A)
\end{aligned}
$$

Therefore by i.h.:

$\#_{\min }([\Gamma] \Rightarrow\langle\rangle A) \leq 0 \leq \#_{\max }([\Gamma] \Rightarrow\langle\rangle A)$

\subsection{Exponentials}

- $\frac{\Delta(A) \Rightarrow D}{\Delta(! A) \Rightarrow D} ! L$

For atoms,

$\#_{\min , P}(\Delta(! A) \Rightarrow D)=$

$\#_{\min , P}^{\circ}(D)-\#_{\max , P}^{\bullet}(\Delta)-\#_{\max , P}^{\bullet}(! A)=$ $\#_{\min , P}^{\circ}(D)-\#_{\max , P}^{\bullet}(\Delta)-X\left(\#_{\max , P}^{\bullet}(A)\right) \leq$ $\#_{\min , P}^{\circ}(D)-\#_{\max , P}^{\bullet}(\Delta)-\#_{\max , P}^{\bullet}(A)=$ $\#_{\min , P}(\Delta(A) \Rightarrow D) \leq 0$ i.h.

For brackets, 
$\#_{\min ,[]}(\Delta(! A) \Rightarrow D)=$

$\#_{\min ,[]}^{\circ}(D)-\#_{\max ,[]}^{\bullet}(\Delta)-\#_{\max ,[]}^{\bullet}(! A)=$ $\#_{\min ,[]}^{\circ}(D)-\#_{\max ,[]}^{\bullet}(\Delta)-\top \leq$

$\#_{\min ,[]}^{\circ}(D)-\#_{\max ,[]}^{\bullet}(\Delta)-\#_{\max ,[]}^{\bullet}(A)=$

$\#_{\min ,[]}(\Delta(A) \Rightarrow D) \leq 0$ i.h.

For atoms and brackets,

$\#_{\max , Q}(\Delta(! A) \Rightarrow D)=$

$\#_{\max , Q}^{\circ}(D)-\#_{\min , Q}^{\bullet}(\Delta)-\#_{\min , Q}^{\bullet}(! A)=$

$\#_{\max , Q}^{\circ}(D)-\#_{\min , Q}^{\bullet}(\Delta)-Y\left(\#_{\min , Q}^{\bullet}(A)\right) \geq$

$\#_{\max , Q}^{\circ}(D)-\#_{\min , Q}^{\bullet}(\Delta)-\#_{\min , Q}^{\bullet}(A)=$

$\#_{\max , Q}(\Delta(A) \Rightarrow D) \geq 0$ i.h.

- $\frac{! A_{1}, \ldots, ! A_{n} \Rightarrow A}{! A_{1}, \ldots, ! A_{n} \Rightarrow ! A} ! R$

For atoms,

$\#_{m, P}\left(! A_{1}, \ldots, ! A_{n} \Rightarrow ! A\right)=$

$\#_{m, P}^{\circ}(! A)-\#_{\bar{m}, P}^{\bullet}\left(! A_{1}, \ldots, ! A_{n}\right)=$

$\#_{m, P}^{\circ}(A)-\#_{\bar{m}, P}^{\bullet}\left(! A_{1}, \ldots, ! A_{n}\right)=$

$\#_{m, P}\left(! A_{1}, \ldots, ! A_{n} \Rightarrow A\right)$

For brackets,

$\#_{m,[]}\left(! A_{1}, \ldots, ! A_{n} \Rightarrow ! A\right)=$

$\#_{m,[]}^{\circ}(! A)-\#_{\bar{m},[]}^{\bullet}\left(! A_{1}, \ldots, ! A_{n}\right)=$

$\#_{m,[]}^{\circ}(A)-\#_{\bar{m},[]}^{\bullet}\left(! A_{1}, \ldots, ! A_{n}\right)=$

$\#_{m,[]}^{\circ}(A)-\#_{\bar{m},[]}^{\bullet}\left(! A_{1}, \ldots, ! A_{n}\right)=$

$\#_{m,[]}\left(! A_{1}, \ldots, ! A_{n} \Rightarrow A\right) \geq 0$ i.h.

- $\frac{\Delta\left(! A_{0}, \ldots, ! A_{n},\left[! A_{0}, \ldots, ! A_{n}, \Gamma\right]\right) \Rightarrow D}{\Delta\left(! A_{0}, \ldots, ! A_{n}, \Gamma\right) \Rightarrow D} ! C$

For atoms,

$\#_{\min }\left(\Delta\left(! A_{0}, \ldots, ! A_{n}, \Gamma\right) \Rightarrow D\right)=$ $\#_{\min }^{\mathrm{O}}(D)-\#_{\max }^{\bullet}(\Delta, \Gamma)$

$\min _{-\#_{\max }^{\bullet}}\left(! A_{0}\right)-\cdots-\#_{\max }^{\bullet}\left(! A_{n}\right)=$ $\#_{\min }^{\circ}(D)-\#_{\max }^{\bullet}(\Delta, \Gamma)$

$-X\left(\#_{\max }^{\bullet}\left(A_{0}\right)\right)-\cdots-X\left(\#_{\max }^{\bullet}\left(A_{n}\right)\right) \leq$ $\#_{\min }^{\circ}(D)-\#_{\max }^{\bullet}(\Delta,[\Gamma])-$

$X\left(\#_{\max }^{\bullet}\left(A_{0}\right)\right)-\cdots-X\left(\#_{\max }^{\bullet}\left(A_{n}\right)\right)-$

$X\left(\#_{\max }^{\bullet}\left(A_{0}\right)\right)-\cdots-X\left(\#_{\max }^{\bullet}\left(A_{n}\right)\right)=$

$\#_{\min }\left(\Delta\left(! A_{0}, \ldots, ! A_{n}, \mid\right.\right.$

$\left.\left.\left[! A_{0}, \ldots, ! A_{n}, \Gamma\right]\right) \Rightarrow D\right) \leq 0$

For brackets, $\#_{\min }\left(\Delta\left(! A_{0}, \ldots, ! A_{n}, \Gamma\right) \Rightarrow D\right)=$

$\#_{\min }^{\mathrm{O}}(D)-\#_{\max }^{\bullet}(\Delta, \Gamma)$

$\min _{\max }^{\bullet}\left(! A_{0}\right)-\cdots-\#_{\max }^{\bullet}\left(! A_{n}\right)=$

$\#_{\min }^{\circ}(D)-\#_{\max }^{\bullet}(\Delta, \Gamma)-\mathrm{T}-\cdots-\mathrm{\top} \leq$

$\#_{\min }^{O}(D)-\#_{\max }^{\bullet}(\Delta,[\Gamma])-\top-\cdots-\top-$

$\mathrm{\top}-\cdots-\mathrm{\top}=$

$\#_{\min }\left(\Delta\left(! A_{0}, \ldots, ! A_{n}\right.\right.$,

$\left.\left.\left[! A_{0}, \ldots, ! A_{n}, \Gamma\right]\right) \Rightarrow D\right) \leq 0$

And for atoms and brackets,

$\#_{\max }\left(\Delta\left(! A_{0}, \ldots, ! A_{n}, \Gamma\right) \Rightarrow D\right)=$ $\#_{\text {max }}^{\circ}(D)-\#_{\min }^{\bullet}(\Delta, \Gamma)$

$-\#_{\min }^{\bullet}\left(! A_{0}\right)-\cdots-\#_{\min }^{\bullet}\left(! A_{n}\right)=$

$\#_{\max }^{\circ}(D)-\#_{\min }^{\bullet}(\Delta, \Gamma)$

$-Y\left(\#_{\min }^{\bullet}\left(A_{0}\right)\right)-\cdots-Y\left(\#_{\min }^{\bullet}\left(A_{n}\right)\right) \geq$

$\#_{\max }^{\circ}(D)-\#_{\min }^{\bullet}(\Delta,[\Gamma])-$

$Y\left(\#_{\min }^{\bullet}\left(A_{0}\right)\right)-\cdots-Y\left(\#_{\min }^{\bullet}\left(A_{n}\right)\right)-$

$Y\left(\#_{\min }^{\bullet}\left(A_{0}\right)\right)-\cdots-Y\left(\#_{\min }^{\bullet}\left(A_{n}\right)\right)=$

$\#_{\max }\left(\Delta\left(! A_{0}, \ldots, ! A_{n}\right.\right.$,

$\left.\left.\left[! A_{0}, \ldots, ! A_{n}, \Gamma\right]\right) \Rightarrow D\right) \geq 0$

$$
\text { - } \frac{\Delta(A) \Rightarrow D \quad \Delta(A, A) \Rightarrow D \ldots}{\Delta(? A) \Rightarrow D} ? L
$$

For atoms and brackets,

$\#_{\min }(\Delta(? A) \Rightarrow D)=$

$\#_{\min }^{\mathrm{O}}(D)-\#_{\max }^{\bullet}(\Delta)-\#_{\max }^{\bullet}(? A)=$

$\#_{\min }^{\mathrm{O}}(D)-\#_{\max }^{\bullet}(\Delta)-X\left(\#_{\max }^{\bullet}(A)\right) \leq$

$\#_{\min }^{\mathrm{O}}(D)-\#_{\max }^{\bullet}(\Delta)-\#_{\max }^{\bullet}(A)=$

$\#_{\min }(\Delta(A) \Rightarrow D) \leq 0$ i.h.

And

$\#_{\max }(\Delta(? A) \Rightarrow D)=$

$\#_{\max }^{\circ}(D)-\#_{\min }^{\bullet}(\Delta)-\#_{\min }^{\bullet}(? A)=$

$\#_{\max }^{\circ}(D)-\#_{\min }^{\bullet}(\Delta)-Y\left(\#_{\min }^{\bullet}(A)\right) \geq$

$\#_{\text {max }}^{\circ}(D)-\#_{\text {min }}^{\bullet}(\Delta)-\#_{\min }^{\bullet}(A)=$

$\#_{\max }(\Delta(A) \Rightarrow D) \geq 0$ i.h.

- $\frac{\Gamma \Rightarrow A}{\Gamma \Rightarrow ? A} ? R$

For atoms and brackets,

$\#_{\min }(\Gamma \Rightarrow ? A)=$

$\#_{\text {min }}^{\mathrm{O}}(? A)-\#_{\text {max }}^{\bullet}(\Gamma)=$

$Y\left(\#_{\text {min }}^{O}(A)\right)-\#_{\text {max }}^{\bullet}(\Gamma) \leq$

$\#_{\min }^{\circ}(A)-\#_{\max }^{\bullet}(\Gamma)=$

${ }_{\min }^{\mathrm{O}}(\Gamma \Rightarrow A)$ 
And,

$$
\begin{aligned}
& \#_{\max }(\Gamma \Rightarrow ? A)= \\
& \#_{\max }^{\circ}(? A)-\#_{\text {min }}^{\bullet}(\Gamma)= \\
& X\left(\#_{\max }^{\circ}(A)\right)-\#_{\min }^{\bullet}(\Gamma) \geq \\
& \#_{\max }^{\circ}(A)-\#_{\min }^{\bullet}(\Gamma)= \\
& \#_{\max }^{\circ}(\Gamma \Rightarrow A) \\
& \bullet \frac{\Gamma \Rightarrow A \quad \Delta \Rightarrow ? A}{\Gamma, \Delta \Rightarrow ? A} ? M
\end{aligned}
$$

For atoms and brackets,

$$
\begin{aligned}
& \#_{\min }(\Gamma, \Delta \Rightarrow ? A)= \\
& \#_{\text {min }}^{\circ}(? A)-\#_{\text {max }}^{\bullet}(\Gamma)-\#_{\text {max }}^{\bullet}(\Delta)= \\
& Y\left(\#_{\min }^{\mathrm{O}}(A)\right)-\#_{\max }^{\bullet}(\Gamma)-\#_{\max }^{\bullet}(\Delta) \leq \\
& Y\left(\#_{\text {min }}^{\circ}(A)\right)+\#_{\text {min }}^{\circ}(A)-\#_{\text {max }}^{\bullet}(\Gamma)-\#_{\text {max }}^{\bullet}(\Delta)= \\
& \underbrace{\underbrace{\#_{\min }^{\circ}(A)-\#_{\max }^{\bullet}(\Gamma)}_{\text {min }}+\underbrace{\#_{\min }^{\circ}(? A)-\#_{\max }^{\bullet}(\Delta)}_{\leq 0 \text { i.h. }}}_{\leq 0 \text { i.h. }} \\
& \underbrace{\underbrace{\circ}_{\max }(A)-\#_{\min }^{\bullet}(\Gamma)}_{\geq 0 \text { i.h. }}+\underbrace{\#_{\max }^{\circ}(? A)-\#_{\min }^{\bullet}(\Delta)}_{\geq 0 \text { i.h. }}
\end{aligned}
$$

\section{Evaluation}

By way of evaluation of the exponential count invariance we compared the performance of Cat$\log 2$ (version f8.1) which uses only multiplicative and additive count invariance with CatLog version $\mathrm{j} 2$ which uses in addition the exponential invariance, ${ }^{1}$ both running under XGP Prolog on a MacBook Air. The lexicon was the same in both cases.

We timed individually the exhaustive parsing of the expressions in Figure 3. Thus, for the sentence a:

\section{(10) $[$ john] + likes+the+man : $S f$}

there is the result of lexical lookup:

$$
\text { (11) } \begin{aligned}
& {[\square t(s(m)): j], } \\
& \square((\langle\rangle \exists g N t(s(g)) \backslash S f) / \exists a N a): \\
& \lambda A \lambda B(\text { Pres }((\text { like } A) B)),
\end{aligned}
$$

\footnotetext{
${ }^{1}$ The engines are otherwise the same.
}

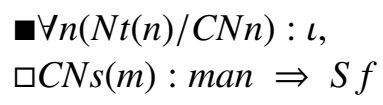

Note that these types include, in addition to the Lambek connectives, normal modalities for intensionality - for rigid designators and $\square$ for semantically active intensionality - and firstorder quantifiers for features; these connectives are transparent to count-invariance. There is the derivation given in Figure 4, which delivers logical form:

\section{(12) $($ Pres $((` l i k e(\iota `$ man $)) j))$}

CatLog proceeds by focalised proof search (Morrill and Valentín, 2015b). The focusing discipline considerably reduces redundancy in the sequent proof search space. The focusing discipline comprises alternating phases of invertible rule application and focalised non-invertible rule application. The boxes in the derivations mark the focused types in focused rule application, i.e. the active types decomposed by non-invertible rule applications. The focusing constrains proof search but in displaying proofs the boxes are limited to this decorative role.

For the sentence d:

\section{(13) $\operatorname{man}+[[$ that $+[$ john $]+$ likes $]]: C N s(m)$}

there is the lexical lookup:

$$
\text { (14) } \begin{aligned}
& \square C N s(m): \text { man, } \\
& {\left[\left[\mathbf { \square } \forall n \left([]^{-1}[]^{-1}(C N n \backslash C N n) / \mathbf{\square}((\langle>N t(n) \sqcap\right.\right.\right.} \\
& ! \square N t(n)) \backslash S f)): \lambda A \lambda B \lambda C[(B C) \wedge(A C)], \\
& {[\square N t(s(m)): j], } \\
& \square((\langle\rangle \exists N t(s(g)) \backslash S f) / \exists a N a): \\
& \lambda D \lambda E(\text { Pres }((\text { like } D) E))]] \Rightarrow C N s(m)
\end{aligned}
$$

Note that these types include also an additive and an exponential which are subject to the countinvariance presented in this paper. There is the derivation given in Figure 5. This uses stoups for the sequent derivation with exponentials (Girard, 2011), (Morrill, 2017). It delivers the logical form:

\section{(15) $\lambda C\left[\left({ }^{(} \operatorname{man} C\right) \wedge\left(\right.\right.$ Pres $\left(\left({ }^{`}\right.\right.$ like $\left.\left.\left.\left.C\right) j\right)\right)\right]$}

The resulting times in seconds were as follows: 

a. John likes the man.
b. Mary thinks that John likes the man.
c. Suzy believes that Mary thinks that John likes the man.
d. man that John likes
e. man that Mary thinks that John likes
f. man that Suzy believes that Mary thinks that John likes
g. Mary talks and Bill sings.
h. John walks Mary talks and Bill sings.
i. Suzy laughs John walks Mary talks and Bill sings.
j. Bill walks Suzy laughs John walks Mary talks and Bill sings.
k. Suzy talks Bill walks Suzy laughs John walks Mary talks and Bill sings.
1. John sings Suzy talks Bill walks Suzy laughs John walks Mary talks and Bill sings.

Figure 3: Example sentences

$$
\begin{aligned}
& C N s(m) \Rightarrow C N s(m)
\end{aligned}
$$

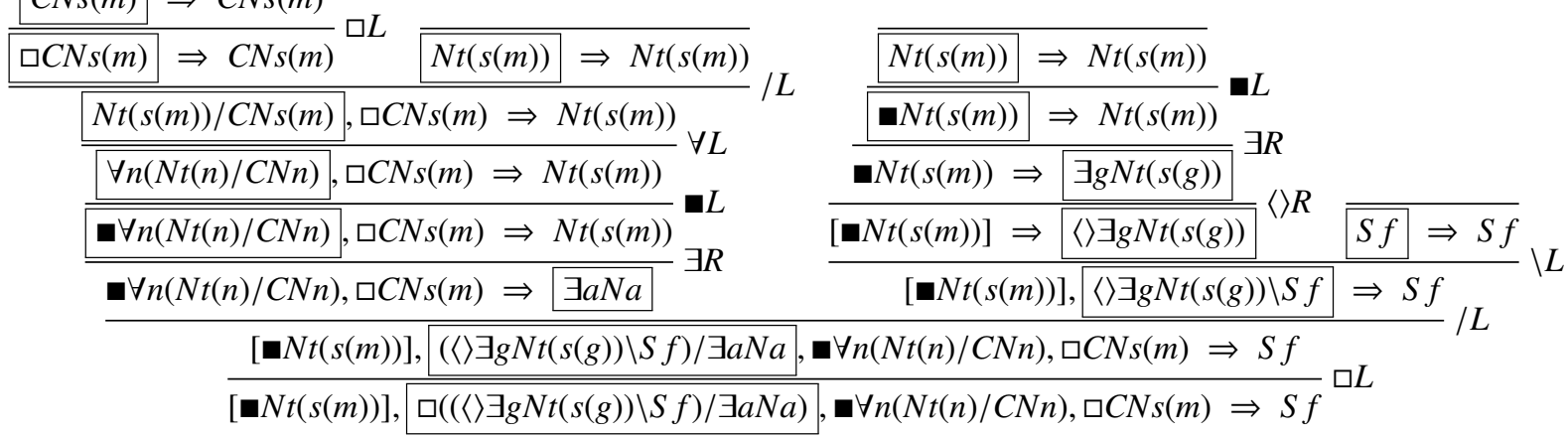

Figure 4: Derivation of example a

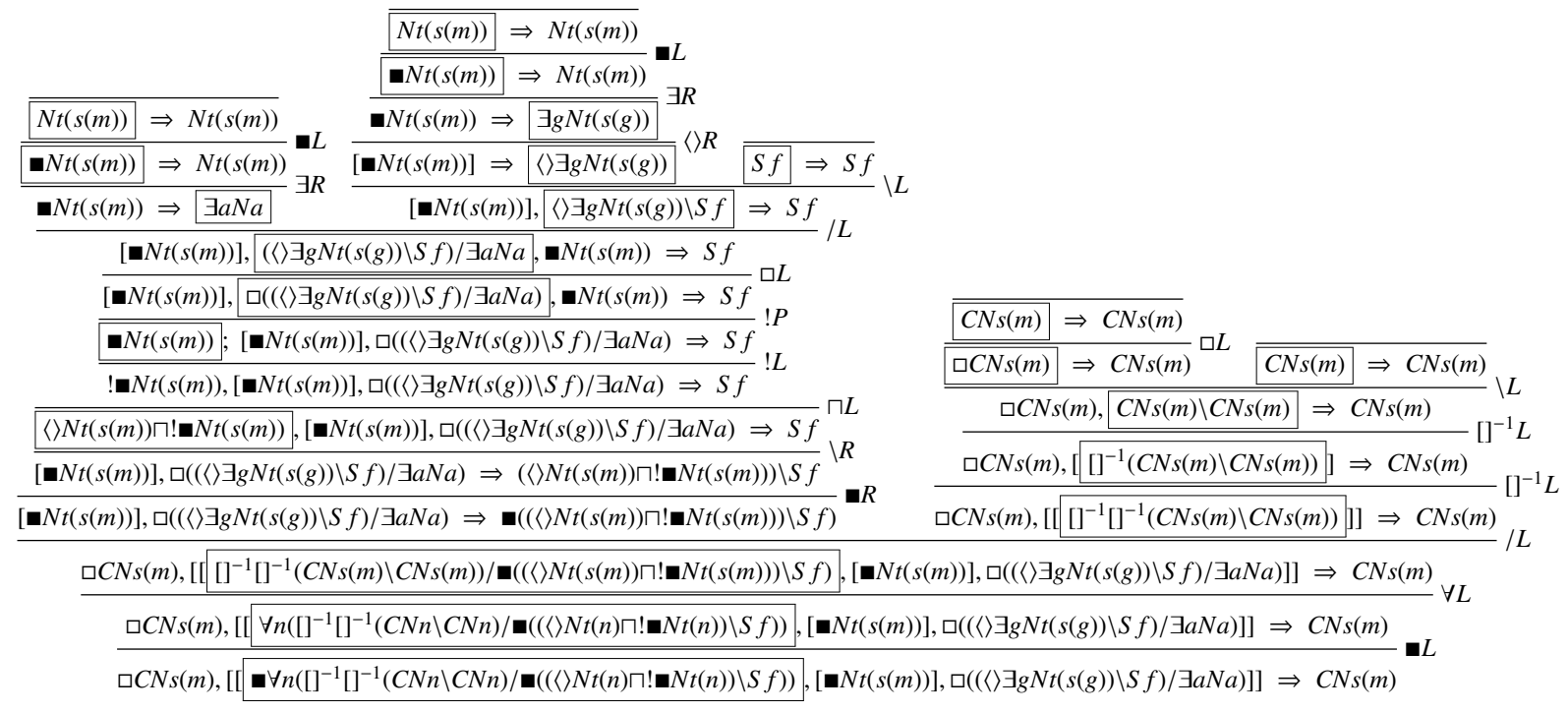

Figure 5: Derivation of example d 
(16)

\begin{tabular}{c|c|c} 
& $\begin{array}{c}\text { f8.1 } \\
\text { (no exp. inv) }\end{array}$ & $\begin{array}{c}\mathrm{j} 2 \\
\text { (exp. inv) }\end{array}$ \\
\hline a. & 1 & 1 \\
b. & 2 & 2 \\
c. & 40 & 6 \\
\hline d. & 2 & 2 \\
e. & 4 & 4 \\
f. & 265 & 6 \\
\hline g. & 1 & 1 \\
h. & 2 & 1 \\
i. & 2 & 1 \\
j. & 2 & 2 \\
k. & 2 & 3 \\
l. & 2 & 4
\end{tabular}

We see that for the longer, third, example of a$\mathrm{c}$ there is a speedup. This is mostly in the time taken to discard inappropriate lexical choices (e.g. that is lexically ambiguous between a complementiser and a relative pronoun): to show that there are no further analyses. The examples d-f involve the universal exponential in a relative pronoun roughly of the form $(C N \backslash C N) /((\langle\rangle N \sqcap ! N) \backslash S)$; the (semantically inactively) additively conjoined hypothetical subtypes are for subject relativisation and object relativisation respectively (Morrill, 2017). Again we see a considerable speedup with exponential count invariance in the longer third case. The examples g-l involve the existential exponential in a coordinator assignment roughly of the form $\left(? S \backslash[]^{-1}[]^{-1} S\right) / S$ to obtain the iteration. Here there is no gain from the exponential type invariance; the overhead causes a slowdown.

For the minicorpus examples of the Montague Test (Morrill and Valentín, 2016a), and for the full CatLog2 corpus (Montague minicorpus, typical categorial examples, discontinuity examples, relativisation and coordination examples, and some Scripture) the parsing times in seconds were:

\begin{tabular}{l|c|c} 
& $\mathrm{f} 8.1$ & $\mathrm{j} 2$ \\
\hline Montague Test. & 37 & 32 \\
CatLog2 corpus & 826 & 643
\end{tabular}

We interpret the experiment as showing that the pruning of the search space of count-invariance including exponentials outweighs the overhead that it engenders: it delivers a speedup of around $20 \%$.

\section{Acknowledgements}

SK supported by RFBR. GM supported by an ICREA Academia 2012. GM and OV supported by MINECO TIN2014-57226-P (APCOM).

\section{References}

K. Ajdukiewicz. 1935. Die syntaktische Konnexität. Studia Philosophica 1:1-27. Translated in S. McCall, editor, 1967, Polish Logic: 1920-1939, Oxford University Press, Oxford, 207-231.

J.-Y. Girard. 1987. Linear logic. Theoretical Computer Science 50:1-102.

J.-Y. Girard. 2011. The Blind Spot. European Mathematical Society, Zürich.

J. Lambek. 1958. The mathematics of sentence structure. American Mathematical Monthly 65:154-170.

G. Morrill. 2012. CatLog: A Categorial Parser/Theorem-Prover. In LACL 2012 System Demonstrations. Nantes, Logical Aspects of Computational Linguistics 2012, pages 13-16.

G. Morrill. 2017. Grammar logicised: relativisation. Linguistics and Philosophy 40(2):119-163.

G. Morrill and O. Valentín. 2015a. Computational Coverage of TLG: Nonlinearity. In M. Kanazawa, L.S. Moss, and V. de Paiva, editors, Proceedings of NLCS'15. Third Workshop on Natural Language and Computer Science. Kyoto, volume 32 of EPiC, pages 51-63.

G. Morrill and O. Valentín. 2015b. MultiplicativeAdditive Focusing for Parsing as Deduction. In I. Cervesato and C. Schürmann, editors, First International Workshop on Focusing, LPAR 2015. Suva, Fiji, number 197 in EPTCS, pages 29-54.

G. Morrill and O. Valentín. 2016a. Computational coverage of Type Logical Grammar: The Montague Test. In C. Piñón, editor, Empirical Issues in Syntax and Semantics, Colloque de Syntaxe et Sémantique à Paris, Paris, volume 11, pages 141-170.

G. Morrill and O. Valentín. 2016b. On the Logic of Expansion in Natural Language. In M. Amblard, P. de Groote, S. Pogodalla, and C. Retoré, editors, Proceedings of Logical Aspects of Computational Linguistics, LACL'16, Nancy. Springer, Berlin, volume 10054 of LNCS, FoLLI Publications on Logic, Language and Information, pages 228-246.

O. Valentín, D. Serret, and G. Morrill. 2013. A Count Invariant for Lambek Calculus with Additives and Bracket Modalities. In G. Morrill and M.-J. Nederhof, editors, Proceedings of Formal Grammar 2012 and 2013. Springer, Berlin, volume 8036 of Springer LNCS, FoLLI Publications in Logic, Language and Information, pages 263-276.

J. van Benthem. 1991. Language in Action: Categories, Lambdas, and Dynamic Logic. Number 130 in Studies in Logic and the Foundations of Mathematics. North-Holland, Amsterdam. 